\title{
Una mirada interseccional a las prácticas de salud en Aysén. Procesos de racialización en Chile
} An intersectional view of health practices in Aysén. Racialization processes in Chile

\author{
Caterine Galaz \\ Universidad de Chile \\ Pastor Cea \\ Universidad de Aysén
}

\author{
Daniela Molina \\ Daniela Castro \\ María José Ortega \\ Universidad de Chile
}

\section{Resumen}

Chile ha diversificado su población migrante a partir de la llegada de personas de América Latina y, a pesar de no ser estadísticamente significativo, esto ha generado una proliferación de imaginarios sociales negativos, afianzados institucionalmente a través de enfoques restrictivos hacia colectivos extranjeros. El racismo institucional se plasma en políticas, discursos y prácticas, ubicando en posiciones de desigualdades a algunos grupos migrantes. A través de una investigación cualitativa llevada a cabo en la XI región de Aysén (Chile), y por medio de un análisis interseccional, presentamos las maneras en que se articulan desigualdades como la procedencia nacional, la clase y el género, en la atención en salud pública. Los resultados muestran la existencia de prácticas racistas institucionales e imaginarios de "otredad" que les subyacen; las formas en que opera un racismo institucional a partir de discriminaciones explícitas; y las agencias y resistencias llevadas a cabo por personas racializadas.

Palabras clave: Racismo institucional; Sistema de salud; Interseccionalidad; Agencia

\begin{abstract}
Chile diversified the migrant population, starting with the arrival of people from Latin America. This, still far from being statistically significant, has generated a proliferation of negative social imaginaries, institutionally consolidated through restrictive approaches towards certain foreign groups.

Institutional racism is reflected in policies and practices, placing migrant bodies in positions of inequality. Through qualitative research carried out in the XI region of Aysén (Chile), and through an intersectional analysis, we present the ways in which different inequalities - such as national origin, class and gender are articulated - in access and care in public health. The results show the existence of institutional racist practices and the imaginaries of "otherness"; the ways in which institutional racism operates based on explicit discrimination; and the agencies and resistance carried out by racialized people.
\end{abstract}

Keywords: Institutional racism; Health system; Intersectionality; Agency 


\section{ANTECEDENTES}

El aumento de población inmigrante en Chile ha supuesto diversos desafíos para el Estado. Sin embargo, la respuesta política ha dejado en evidencia prácticas y discursos racistas que criminalizan, racializan y discriminan diferenciadamente a ciertos colectivos de la población migrante, ubicándolos en espacios sociosimbólicos de subalternidad (Dube, 2001). Estos discursos provienen desde la misma institucionalidad, por lo tanto, poseen una carga no sólo simbólica, sino también una eficacia social (Maffesoli, 2003) solidificando comportamientos xenófobos y racistas.

La migración en Chile tiene larga data, acentuándose en los años 90 producto del proceso de restauración y recomposición democrática (Núñez y Torres, 2007). Hoy en día residen en Chile 1492522 personas extranjeras, provenientes principalmente de Venezuela $(30,5 \%)$, Perú $(15,8 \%)$, Haití $(12,5 \%)$, Colombia $(10,8 \%)$ y Bolivia (8\%) (INE-DEM, 2020), de las cuales $51 \%$ son hombres y $49 \%$ mujeres (INE-DEM, 2020).

Un 65,1\% de las personas migrantes está cubierta por el Fondo Nacional de Salud (FONASA), un $14,7 \%$ por Instituciones de Salud Previsional (ISAPRE) y un 2,8\% por ningún servicio (ni privado ni público). Desde el 2017 Chile cuenta con una Política de salud migrante, basada en una perspectiva de Derechos Humanos, considerando determinantes sociales de la salud, un enfoque de género e interculturalidad, y que establece principios como la participación ciudadana, la no discriminación, la cohesión social y la igualdad.

No obstante, la población migrante presenta una mayor falta de consulta, de cobertura e insatisfacción de necesidades sanitarias (Cabieses y Oyarte, 2020; Galaz y Cea-Merino, 2020). La atención de salud de esta población cuenta con limitaciones de acceso que operan en un nivel estructural, como son las barreras culturales y políticas; a un nivel institucional, con barreras organizacionales y de prestación de servicios; $y$, a un nivel individual, a raíz de características específicas de las personas migrantes y de los/as proveedores/as de servicios (Chepo et al., 2019).

En este artículo analizamos cómo se articula el racismo institucional en el ámbito de la salud con otras desigualdades como el clasismo y el sexismo, dejando a ciertos colectivos migrantes en situaciones de vulnerabilidad. La investigación se llevó a cabo en la región de Aysén, la cual ha experimentado un aumento importante a pesar de su baja conectividad con el resto del territorio. En esta región la migración latinoamericana se ha incrementado en el último tiempo, estimándose al 2019 un total de 3988 personas extranjeras residentes, lo cual representa un aumento absoluto de 620 personas y un alza relativa de 
$18,4 \%$ respecto a lo estimado para 2018, predominando la población migrante argentina con un $49,4 \%$, Colombia con $16,9 \%$, Perú con $4,5 \%$ y E.E.U.U con un $4.1 \%$ (INE- DEM, 2020).

Por otro lado, cabe tener en cuenta que los estudios sobre racismo desde la psicología en Latinoamérica principalmente se han enfocado en las consecuencias psicológicas de la discriminación en colectivos afrodescendientes e indígenas (Pineda, 2018). Esther Pineda (2018) explora las consecuencias de la discriminación racial en personas afrodescendientes y Alfonso Urzúa et al., (2019) se enfocan en el estrés producto de la aculturación de inmigrantes. Para Marizete Gouveia y Valeska Zanello (2019) la relación entre la psicología y el racismo no ha sido lo suficientemente abordada, lo que produce que los/as profesionales de la psicología no consideren la noción de "raza" como una categoría a considerar. David Pavón Cuellar (2019) analiza el papel de la psicología dominante en la reproducción de la violencia estructural -incluyendo el racismo- por el ocultamiento de causas de tipo económico, social y político a factores psíquicos, personales e interpersonales. En ese sentido, propone evitar la reducción psicologizante e individualizantes de fenómenos sociales.

Consideramos en este artículo, relevante desde una psicología social crítica y feminista (García-Dauder, 2010, Kitzinger, 2003; Wilkinson, 1991), incluir una perspectiva interseccional, ya que este enfoque entra en tensión con corrientes tradicionales al relevar las dimensiones estructurales de las opresiones interconectadas (Cea-Merino y Montenegro, 2014; Montenegro et al., 2011), permitiendo análisis más complejos de las desigualdades sociales. Como insisten Dauder García Dauder (2010) y Sue Wilkinson (1991), se requiere politizar a la psicología precisamente para evitar que se realicen análisis acordes a una psicología dominante que puede servir a la reproducción de formas de dominación.

\section{MARCO TEÓRICO: RACISMO INSTITUCIONAL E INTERSECCIONALIDAD}

Teun Van Dijk (2006) enfatiza que el concepto de "raza" fue desarrollado teóricamente por sectores científicos privilegiados, de la misma manera que la superioridad racial, lo que dio sustento al colonialismo, la eugenesia, la segregación, el holocausto, el apartheid y la limpieza étnica en diferentes contextos sociales. Para Michael Foucault (1975/1992) el poder de los estados occidentales y el discurso biologicista se apoyó en una contrahistoria para llevar a cabo las bases teóricas del racismo, lo que permitió la hegemonía de un tipo de sociedad a través de dispositivos disciplinarios. Asimismo, el relativismo cultural contribuyó a la caída del determinismo biológico, pero, a la vez, le dio pie a postulados diferenciadores excluyentes culturalistas entre grupos sociales (Ga- 
laz, 2009). Esta mirada ha alimentado posturas racistas basadas en enfoques culturalistas o, como destaca Teresa San Román (1996), al surgimiento de un racismo social sin raza.

El racismo emerge como una construcción social generada con el fin de perpetuar una dominación, en donde "el proyecto modernizador usó a la 'raza' como categoría de clasificación y de diferenciación social” (Tijoux, 2014, p. 1). Así, estaríamos ante una estructura racista "que se niega a declarar expresamente su tendencia a discriminar al negro y se refugia en sobreentendidos, supuestos y afirmaciones implícitas" (Javaloy, 1994, p. 23).

En ello, el Estado se hace parte del racismo, generando reproducciones, dispositivos y performance de exclusión de ciertos colectivos. Angela Pace y Marluce Lima (2011) sugieren que el racismo institucional fue acuñado para denunciar cómo se manifestaba en las estructuras de organización de la sociedad, con el fin de describir los intereses, acciones y mecanismos de exclusión establecidos por los grupos racialmente dominantes. El racismo institucional se manifestaría en prácticas discursivas de las elites, expresadas en posiciones políticas, discursos periodísticos, discursos técnicos, normas y lenguaje burocrático y de la administración social (Van Dijk, 2006). Pero estas prácticas discursivas no sólo son ideas, sino que tendrían una eficacia social (Maffesoli, 2003) porque se materializan en prácticas, actitudes, comportamientos y violencias directas e indirectas hacia algunos colectivos (Galaz, 2009). Para Suzana Kalckmann et al. (2007) el concepto de racismo institucional refiere a aquel fracaso colectivo de las organizaciones y a diferenciar a las personas por su color, cultura u origen. Éste puede traspasar la institucionalidad al mostrar prejuicio involuntario, ignorancia, negligencia y estereotipación racista, que causa desventajas a las personas de minorías.

En este artículo comprenderemos el racismo institucional como el resultado del poder gubernamental expresado en ciertos dispositivos sociales que se llevan a cabo por medio de una regularización biopolítica y sobre un disciplinamiento de los cuerpos (Foucault, 1975/1992), situando a ciertas personas o grupos racializados en una condición de desventaja en el goce de derechos.

Por otro lado, Patricia Hill Collins (2000) plantea a la interseccionalidad ${ }^{1}$ como un paradigma estructural para evidenciar la articulación entre diversos siste-

${ }^{1}$ Kimberlé Crenshaw (1989) acuñó el concepto para visibilizar la discriminación laboral específica que cruzaron mujeres negras en EEUU. Sin embargo, esta inquietud ha estado presente desde muchos años antes en luchas de movimientos feministas y abolicionistas de la esclavitud. Es importante considerar a Sojourner Truth y su discurso ¿Acaso no soy mujer? (Brah, 2013). En las últimas décadas, se ha complejizado la noción desde propuestas negras antirracistas (Hill Collins, 2000; hooks, 1984), chicanas lésbicas (Anzaldúa, 2007; Moraga, 2000) y post y decoloniales (Lugones, 2008; Mohanty, 2003). 
mas de dominación como el racismo en relación con el sexismo, el clasismo y la heteronorma, entre otros. Esta perspectiva nos permite analizar procesos complejos donde no sólo opera una única categoría de diferencia, sino que se articulan para establecer restricciones a determinados colectivos sociales que socialmente son situados en esos cruces de desigualdades (Avtar Brah; 2013; Hill Collins y Bilge, 2016). Tal como sugiere Mary Nash (2006) no se trata de perspectivas multiplicativas de las categorías de diferenciación, las cuales incurren en la identificación de un sujeto doble o triplemente oprimido (Montenegro, 2014), sino más bien en la complejización del cruce de estas diferenciaciones estructurales.

Como plantea Danielle Kergoat, las relaciones sociales tejen una trama y por tanto son consustanciales, es decir, que coexisten y se impulsan a la vez dentro de la matriz societal (2003). Para este artículo es importante poder dar cuenta de cómo diferentes categorías, al converger, dejan en evidencia discriminaciones más complejas, puesto que se reconocen desigualdades estructurales que posibilitan múltiples violencias que viven personas migrantes.

Es importante tener en cuenta a distintos/as autores/as que desde la psicología social y la sociología destacan el potencial crítico de la mirada interseccional para tensionar fenómenos que aparecen naturalizados o sobreentendidos en los estudios sobre migraciones (Carrère y Carrère, 2015; Magliano, 2015). Como señala Carolina Pinto y Nicole Cisternas (2021), el uso de la noción de interseccionalidad no radica solamente en iluminar el entrecruzamiento de diferentes ejes de clasificación social, sino más bien en lograr incorporar uno en específico, importante para la región latinoamericana, el de la "raza".

\section{METOdOLOGíA}

Utilizamos una metodología cualitativa, ya que permite el acercamiento a las comprensiones que las y los propios/as agentes otorgan a los fenómenos sociales (Valles, 1999) y permite analizar, desde interpretaciones situadas, las experiencias y relatos (Sampieri et al., 2004). Llevamos a cabo entrevistas semiestructuradas porque permiten comprender con mayor complejidad lo que quiere expresar el sujeto entrevistado. Estas fueron llevadas a cabo durante un mes en torno a tres perfiles: a) profesionales del área de la salud (funcionarios/as que trabajan en centros de la región de Aysén, lo que aportó un panorama de la dinámica profesional-usuario/a); b) interventores/as en materia de migración en la región, con lo que se observó las matrices vigentes respecto de la población migrante; y c) usuarios/as de diferentes nacionalidades para conocer cómo vivencian la atención en estos servicios de salud. Se entrevistaron a 10 interventores/as (4 trabajadoras sociales, 1 psicólogo, 1 antropólogo, 1 pro- 
fesional de servicio de mujeres, 2 profesionales del departamento de extranjería, 1 directivo de salud regional); 9 profesionales de la salud (6 enfermeras, 2 matronas y 1 doctora); y 11 usuarios/as migrantes ( 3 venezolanos/as, 3 colombianos/as, 3 haitianos/as, 1 brasileña y 1 ecuatoriano).

Consideramos firmas de consentimientos informados y autorizaciones formales de los servicios de salud. El método de selección para la muestra fue teóricamente conducido (Valles, 1999), ya que se buscaba saber las prácticas y discursos en intervención directa con población migrante, seleccionando perfiles específicos que trataran esta relación social. Como criterio de selección de las personas migrantes se buscaron perfiles que coincidieran con los grupos con mayor población residente en el territorio. Llevamos a cabo un análisis de contenido que permitió generar relaciones e inferencias entre los diversos temas analizados con teoría previa (Mayring, 2000, en Cáceres, 2003). Se prestó atención a los discursos sobre la atención de la población migrante, desde una perspectiva interseccional que pone atención a la complejidad inter-categorial (McCall, 2005). Esto quiere decir que se analizaron las formas en que se intersectan distintos ejes de diferencia (color de piel, procedencia nacional, clase y género). A partir de ello, configuramos tres categorías de análisis que se presentan a continuación.

\section{ANÁlISIS: PRÁCTICAS E IMAGINARIOS RACISTAS INSTITUCIONALES}

A través de las entrevistas realizadas vemos cómo el racismo institucional se vincula a cierto racismo simbólico (Javaloy, 1994), de una manera sutil e indirecta. En los siguientes extractos, se registra un discurso que proviene de profesionales que cuentan con un estatus jerárquico (o voz autorizada) asignado por el sistema predominante que les permite transmitir ideas políticas que se convertirán -en última instancia- en verdades irrefutables en el contexto social. Como este racismo no se expresa explícitamente, sino a través de la respetabilidad del hablante, crea imaginarios mediante la acción de la política pública, los que se legitiman en lo cotidiano y que se replican como normas que son compatibles con la tolerancia (Javaloy, 1994), institucionalizando de esta forma prácticas racistas y xenófobas.

Una enfermera comenta acerca del alza del flujo migratorio que está viviendo la región, enfocándolo en particular a la presencia de personas con una fenotipia distinta a la del colectivo nacional. Su discurso busca disfrazar la molestia frente a la residencia de personas afrodescendientes que han llegado al territorio para residir, asumiendo que existiría una diferenciación entre la llegada de extranjeros/as europeos e inmigrantes afrodescendientes: 
Yo creo que el fenómeno migratorio de un tiempo a esta parte ha sido rápido, rápido de quizás de lugares de donde nosotros no estábamos acostumbrados a tener inmigrantes acá... o sea veíamos extranjeros de Alemania, Francia, de Estados Unidos que venían de vacaciones... pero esta gente es diferente, de la raza negra que ha sido, yo creo que mucha gente lo ha tomado como invasivo, porque es mucha y son diferentes a nosotros y vienen a quedarse. (Enfermera 1, entrevista presencial, junio de 2018)

En el extracto se denota cierta aprensión hacia la comunidad migrante que la entrevistada refiere como "raza negra", resaltando la existencia de fuertes diferencias que incluso generarían incomodidad en la población nacional, en última instancia, denostando la negritud (Tijoux, 2014). Cabe señalar que la observación que realiza la profesional respecto a la diferenciación fenotípica de estos cuerpos, se sustenta plenamente en una estructura colonial (Tijoux, 2014).

En la siguiente cita, la racialización se instaura a partir de un clasismoetnocéntrico, en el sentido de que a la población afrodescendiente se le asocia una idea de atraso cultural y pobreza, producto de un supuesto déficit en su educación, junto a la presencia de condiciones sanitarias precarias que se le asignan al colectivo. El país de recepción emerge como un todo homogéneo, sin diferenciaciones internas y con un mayor valor cultural:

Hay aspectos de saneamiento básico que se tienen que reforzar en ellos, o sea por ejemplo muchos de las personas que vienen nunca tuvieron baño adentro de su casa, nunca tuvieron sistema de alcantarillado, nunca tuvieron los elementos necesarios para hacer un aseo correcto y digno, de un baño, de una cocina o manejo de las basuras, etc., entonces en ese aspecto que hay que hacer una educación desde cero... (Trabajadora Social 5, entrevista presencial, junio de 2018)

A consecuencia de esta racialización se valida y legitima una intervención enfocada a la re-educación. El racismo institucional "se refugia en sobreentendidos, supuestos y afirmaciones implícitas" (Javaloy, F. 1994, p. 23). Esto conlleva a justificar un tipo de trato, de atención y de intervención específica hacia la población afrodescendiente, en este caso, a migrantes haitianos que viven en la zona, y que se tornará distinta a las formas clásicas de atención dirigidas al endogrupo (chilenos/as). Se da por entendido que los/as profesionales del área poseen una posición jerárquica superior no sólo por sus conocimientos técnicos, sino que por pertenecer a un grupo social y cultural "más elevado". En consecuencia, actúa un esencialismo que, de acuerdo con Edward Said (2003), no coincide con la multiplicidad identitaria de cualquier colectivo social, sino que resalta las diferencias entre sujetos haciendo hincapié en los conceptos de superioridad versus subordinación (Javaloy, 1994). Por tanto, en 
este ejercicio, se sigue perpetuando actitudes colonialistas como la reeducación de una población a la cual se le considera "carente".

Se va construyendo la imagen de una otredad ajena que representa un problema social que debe ser resuelto -concepción que por lo general se le vincula fuertemente a la población afrodescendiente (Galaz y Montenegro, 2015)-. En el siguiente extracto queda patente como, en el ámbito de salud, la migración haitiana se ha vuelto el centro de atención por antonomasia en los últimos años, ubicándola como una problemática que requiere de programas especiales y diferenciados, y que por sus características, tanto físicas como culturales, deben ser sobre-intervenida:

Lo más problemático para el sistema de salud, que ellos (profesionales) han problematizado la migración haitiana, porque están con una obsesión en la diferencia cultural que partió por el tema del idioma pero que están basadas en cuestiones del color de piel. No se justifica tanta sobre-reacción y sobre-atención cuando no es el colectivo migrante mayoritario... hay una obsesión del sistema de salud porque se adapten los haitianos, perseguirlos para que adhieran a los programas, considerarlos diferentes, extraños, riesgosos, sin considerar que, de otras procedencias, pueden ser igual de diferentes. (Profesional salud, entrevista presencial, junio de 2018)

Tal como lo manifiesta, en cada intervención se van construyendo discursos de extrañeza, los cuales potencian la idea de que el/la migrante debe ser adaptado al nuevo país de residencia a través de una sobre intervención. Esta noción de extrañeza se encuentra muy arraigada a las pautas de acción profesional (Santamaría, 2002), pero se ha fortalecido en el tiempo por la reproducción de prácticas y discursos racistas a nivel social.

La noción de "raza" no es preexistente, sino que surge como una categoría social que contiene ciertas características -en su mayoría negativas- asociadas a un colectivo en particular, denostando atributos como el color de piel, idioma, crianza, entre otras cuestiones, las cuales ponen énfasis en la diferencia cultural. Esta categoría social ampara y perpetúa un racismo institucional que en la práctica cotidiana justificará el trato desigual hacia ciertos colectivos migrantes, en especial afrodescendientes que se atienden en los dispositivos de salud, como se señala:

Los discursos de los profesionales tratantes, acerca de las personas migrantes, están impregnados de discursos sociales que son políticos, institucionales, gubernamentales, administrativos... por ejemplo, normalizadores en relación a ciertas patologías, discriminatorios o de otro tipo que de alguna manera en el fondo trascienden y penetran el espacio clínico y tienen impacto en los diagnósticos de los tratamientos. (Profesional de salud, entrevista presencial, junio de 2018) 
El racismo oculta su intención a través de la negación de la discriminación (Javaloy, 1994), es decir, amparándose bajo discursos moralistas y normalizadores que apuntan al supuesto bienestar común de la población migrante. Sumado a esto, en las intervenciones de salud se pueden observar "escalas de proximidad" (Galaz, 2009) que reflejan la posición jerárquica de los individuos atendidos en estos servicios respecto de la población considerada autóctona -desde nacionales, extranjeros/as, inmigrante hasta afrodescendientes-, lo cual definirá la distancia cultural y fenotípica con el/la profesional de salud y, por ende, la valoración que tendrán durante la atención general hacia ellos/as, como veremos a continuación:

También hay población que dependiendo de dónde yo la pongo, si es más estigmatizable o menos, si, en donde ponemos el estigma en el otro, se generan respuestas: hay gente que, por su raza, por su género, es más estigmatizable; por lo tanto, generan respuestas o relaciones de exclusión. No es lo mismo una mujer rubia que una morena, no es lo mismo una mujer chilena que una indígena, no es lo mismo una europea morena que una blanca, no es lo mismo un hombre a una mujer, y entonces hay prácticas que se institucionalizan, porque hay representaciones que se reproducen en las prácticas institucionales. (Funcionaria de salud, entrevista presencial, junio de 2018)

De esta manera, estas prácticas y discursos que discriminan y estigmatizan el cuerpo migrante, no surgen a priori de las relaciones interpersonales sino que se han instaurado desde lo institucional, generando, en la forma de concebir la salud-enfermedad, discriminaciones que hoy en día quedan más explícitas ante la presencia y residencia de colectivos afrodescendientes.

\section{Racismo y discriminación}

Los imaginarios racializados expuestos no solo quedan como simples ideas mentales o prejuicios, sino que tienen una eficacia simbólica (Maffesoli, 2003), es decir, tienen consecuencias en las interacciones sociales que generan discriminaciones. Estos procesos de diferenciación jerarquizados que se ven en algunos discursos entre nacionales y migrantes racializados/as terminan en exclusión y en un amparo discursivo que justifica esa acción. En la siguiente cita queda patente que parte de los equipos de salud pueden negar la atención a una persona haitiana en el cruce de clase y racialización, dejando en evidencia que no se les considera en igualdad de condiciones respecto de la población nacional:

Hace un tiempo nos llegó un paciente haitiano, obrero, que había sufrido un accidente laboral. Cuando llegó una compañera enfermera me dijo que ella no atendería a ese negro, que lo atendiera yo. Me quedé paralizada. Conozco a esta persona y siempre ha sido buena profesional, no entendía 
por qué decía eso... Falta mucho aún por formar a los mismos profesionales. Es cierto que hablaba poco castellano, pero no era eso... era su color de piel. (Enfermera 2, entrevista presencial, junio de 2018)

Este tipo de conjunción entre racismo y posición de clase no siempre ocurre con personas que son racializadas de sectores empobrecidos, sino también en quienes ostentan una posición más privilegiada económicamente, como pueden ser médicos/as extranjeros/as. En la desconfianza respecto de sus capacidades profesionales y en la solicitud de derivación a médicos/as nacionales, se sustenta también un tipo de discriminación xenofóbica que además nos habla de una visión etnocéntrica al considerar que los/las nacionales se sitúan en una posición de mayor conocimiento y especialización que los/las extranjeros/as:

Hay gente que tiene prejuicios en atenderse con un médico extranjero, o sea, dicen no este, no sé po, es ecuatoriano o cubano y no me quiero atender con él, prefiero esperar y que me atienda un chileno. (Enfermera 5, entrevista presencial, junio de 2018)

No sólo se trata de interacciones interpersonales donde se verifican estas prácticas excluyentes hacia ciertos sujetos racializados, sino también a nivel estructural: son las instituciones también las que construyen en sus prácticas y discursos formas de racismo. En el siguiente extracto se verifica precisamente cómo desde el ministerio de salud se difunden actuaciones que asocian a ciertas enfermedades de transmisión sexual a cuerpos migrantes específicos:

El ministerio hizo una campaña de VIH nacional -yo tuve esos posters aquí pegados, aquí atrás - salía una persona de color y eso causó harta molestia, hasta a uno, yo lo miraba y decía como que dan a entender que solo ellos pueden tener VIH y que uno corre riesgo con esa persona... en realidad el riesgo son las prácticas sexuales, no tiene que ver el tema de la nacionalidad ni el color de piel. Pero ahí estaba, y era obligatorio ponerlo en todos los centros públicos. (Profesional salud 4, entrevista presencial, junio de 2018)

Por un lado, la construcción social del inmigrante "negro" hombre aparece como la figura de otredad por excelencia con este tipo de prácticas, no solo vinculándolo con falta de educación o higiene como se vio anteriormente, sino a través de elementos concretos (posters y campaña de difusión masiva), asociándolo a una "enfermedad". En esta práctica se produce un enemigo biológico desde un racismo institucional que proviene del Estado. Por otro lado, también la construcción homogeneizada de una mujer inmigrante "negra" emerge en prácticas concretas en los servicios, donde se denota cierto trato despectivo en relación con ideas hipersexualizadas, falta de habilidades/capacidades, o 
configuraciones de hipervictimización. Vemos que el trato discriminatorio, dependerá también de la posición de clase de las personas:

Se discrimina por todo, por la clase, el género, el color de piel. Si eres morena vas a ser discriminada porque eres morena, y seguramente después que se den cuenta de que clase social eres, será peor, y de qué país entran distinciones que te hagan vivir más discriminación, alguien que es super rubia o blanca, también sufre discriminación si es de clase baja, no le asegura eso ser menos discriminada, seguramente si no abre la boca, depende de cómo se vista, va a vivir más o menos. Somos un país en que generamos categorías, y eso se ve entre los profesionales también, se fijan en el cuerpo de las personas, en el apellido, en la educación, cómo habla. (Profesional de salud 5, entrevista presencial, junio de 2018)

A pesar de que no se realizan campañas específicas de salud sexual y reproductiva "para" este colectivo, algunos/as funcionarios/as plantean que al final se hace de manera encubierta al convocarles más insistentemente a los servicios, porque persiste la creencia de que la mayor parte de la población que ejerce el comercio sexual es migrante. En este sentido, se ha generado una estigmatización de ciertos colectivos:

Existe una concepción de que las mujeres migradas vienen casi todas a prostituirse, lamentablemente es ese el concepto que, si viene una haitiana, una colombiana, o lo que sea, ellas vienen a prostituirse (...) Se está encima de ellas para que vengan. (Enfermera servicio de salud, entrevista presencial, junio de 2018)

Asimismo, la valoración de ser "mujer madre" varía respecto a si esta tiene nacionalidad chilena o extranjera. En ciertos discursos se cuestionan las habilidades marentales de las extranjeras generando comparaciones etnocéntricas respecto de las capacidades excepcionales de las chilenas. Se idealiza el papel de la mujer "nacional", como si de alguna manera éstas cumplieran con todas las normas sociales esperables para la crianza, posicionándolas en una situación de privilegio respecto de las migrantes:

La forma que tienen de cuidar a sus hijos es distinta porque viene todo de un sistema cultural distinto, por ejemplo, las haitianas o las dominicanas, o sea en otros países los niños prácticamente se crían solos, porque la mamá tiene que salir a trabajar (...) no es como acá que tú mandas a tus hijos al colegio, en la tarde estás con ellos, te preocupas de su higiene personal, de que vayan a sus controles regulares de salud, dentales, allá probablemente no tengan acceso a eso ¿ya? (Trabajadora social 5, entrevista presencial, junio de 2018)

En los relatos recabados en Aysén, se destaca la distinción entre migrante/extranjero-a. Es un territorio con alta afluencia turística en verano y se re- 
calca cierta costumbre a que transiten personas de otras nacionalidades. Se utiliza la distinción entre "migrantes/extranjeros-as" como un método de jerarquización, donde "migrante" adquiere una posición inferior, en especial de mujeres racializadas:

Aquí la gente estaba acostumbrada a las extrajeras porque siempre vienen de Europa, Estados Unidos o Australia y entonces la gente estaba acostumbrada a ese transeúnte extranjero, pero no a este otro tipo de personas migrantes, que son trabajadores muchos, a las mujeres que se dedican a la prostitución, a los que tienen otro color de piel. (funcionaria migración 1, entrevista presencial, junio de 2018)

Vemos la configuración de un "nosotros/as" que tiene características no solo culturalistas, sino también racistas y de clase. Esto lleva a esbozar un principio de prioridad nacional por sobre otros colectivos residentes. Esta construcción homogénea de la procedencia nacional de ciertos colectivos lleva a que se privilegie casi exclusivamente al endogrupo (chilenos/as) en el acceso a derechos sociales.

Es que tú escuchas entre los profesionales y administrativos eso muchas veces 'pero cómo, si somos chilenos, hay que darle preferencia al chileno y no al migrante", o el chileno es tanto o más como el mapuche, a todos por igual, cosas de ese tipo, como que no debieran de darle prioridad a otras personas, menos a los migrantes. (Profesional salud 3, entrevista presencial, junio de 2018)

Las categorías de diferenciación que se han construido históricamente en Chile han adquirido valor y sentido dentro de la sociedad y su institucionalidad gubernamental, posibilitando opresiones múltiples de carácter racial, sexual, de clase y cultural. Por tanto, no solo se trata de relaciones cotidianas, sino que éstas son fruto de una consideración estructural donde el desempeño institucional tiene un rol importante, como se señala:

Hay distintas formas de verlos, algunos son migrantes, pero a otros se les considera extranjeros... eso se traduce en cómo hacemos la política. Estoy convencida de que en la representación que tenemos cotidianamente en nuestra atención tiene que ver con la forma de la política social... las políticas sociales reproducen esas representaciones. (Trabajadora social 2, entrevista presencial, junio de 2018)

\section{Agencia/resistencia migrante}

Muchas personas migrantes son conscientes de la conjunción de los procesos de racialización, nacionalismo, sexismo y clasismo a los que son expuestos/as. Por lo tanto, desarrollan agencias y resistencias específicas para hacerles frente, es decir, acciones que realizan respecto de las dinámicas de poder que cruzan en 
los servicios de salud. Expresión de ello es que algunas mujeres desarrollan estrategias para acudir sólo con ciertos profesionales a los servicios:

Lo que ha pasado era que cuando yo salía de vacaciones la gente no venía (...) eran colombianas. En algún momento yo les pregunté ¿por qué? Porque después llegaba y yo tenía lleno y además me reemplazaban. Me respondían: yo puedo aguantar que a mí me traten mal, alguien que me trata mal de palabra yo me defiendo, pero yo no me puedo defender cuando la gente pone caras, cuando me ponen caras de asco... porque hay que hacer especuloscopías y el trato era displicente, entonces me decían que el servicio debería cerrarse si yo no estaba, porque ellas irían solo conmigo porque era un trato mejor. (Matrona 4, entrevista presencial, junio de 2018)

Se refleja en la cita cómo las mujeres migrantes dejan de asistir a sus controles médicos, dado un mal trato que ellas no tolerarán. Son tácticas de enfrentamiento contra la institución. Tal como sugiere Michel Foucault (1988) son luchas inmediatas, donde las mujeres migrantes critican a quienes o aquello que ejerce acción directa sobre ellas. En muchas situaciones estas mujeres no tienen la posibilidad de acceder a otro espacio de atención en la zona, siendo la negativa a atenderse su única posibilidad de agencia.

Otra dinámica de resistencia es reaccionar buscando otras atenciones de salud. Algunas de las entrevistadas hacen uso de redes de apoyo de sus países de origen, contactando a sus médicos o sus familias para que hablen con algún/a profesional en su país de origen. Lo que realizan es un acto de agencia en el sentido de que, por sus propios medios y posibilidades, buscan una atención y diagnóstico con el que se sientan seguras y conformes.

Llamo a mi familia para que me contacte con el primo que es doctor en Haití. Así estoy más tranquilo porque sé que me dirán lo correcto. Aquí mucho tiempo de espera, o solo me dan paracetamol y para la casa. (Hombre haitiano, entrevista presencial, junio de 2018)

Estas experiencias se profundizan para algunas mujeres migrantes afrodescendientes, ya que están expuestas a enfrentarse a mayores tratos discriminatorios dentro de los servicios de salud, debido a la vivencia de su corporalidad. Reflejo de ello es la experiencia de una mujer residente que explica que prefiere evitar el mal trato acudiendo a videoconferencias con médicos/as de su país para la atención de su hijo. En este caso, la mujer migrante se debe enfrentar a un sistema médico con el cual no está familiarizada y con el que no se siente cómoda, sumándose a ello la responsabilidad de ser buena madre producto de las construcciones sociales hegemónicas por ser mujer migrante:

Siempre he estado en contacto con su pediatra en Venezuela y entonces ya por ahí, su pediatra ya lo conoce, sabe su informe médico, ya lo controla y 
la llamo y ella me dice "ya, dale esto, dale aquello" y entonces ahí yo me las he arreglado... sino tengo que pasar por todo el mal trato aquí, por la desconfianza que veo hacia nosotras como mamás y prefiero no hacerlo. (Mujer venezolana 10, entrevista presencial, junio de 2018)

Según los relatos, el territorio extremo vendría a ser un límite para acceder a derechos de salud integral en esa región de Chile. Además, la clase social exacerba aquello, ya que, para acudir a una "segunda opinión" médica o toma de ciertos exámenes, se debe recurrir a la capital de la región (Coyhaique) o bien a la región Metropolitana, con el alto costo económico de movilidad que esto implica. En algunas zonas de esa región solo hay un centro asistencial de atención primaria, por lo que se deja en evidencia cómo la espacialidad viene a convertirse en una cierta trampa territorial para el ejercicio de los derechos.

Las estrategias autogestionadas de las personas migrantes se vuelven relevantes, y sobre todo las de cooperación intra-grupal como acompañamientos para traducción en caso de personas haitianas, o traspaso de información de "datos médicos" más económicos y con más apertura a la población migrante. Algunas entrevistadas sostienen que los sujetos migrantes que deciden oponerse a ciertas prácticas pueden ser castigados/as desde el sistema de salud, tal como lo plantea la siguiente entrevistada, al hablar de la poca adherencia a programas de salud:

Hay migrantes que para nosotros son invisibles porque nos ponen resistencia, los que oponen resistencia son castigados, dominados, entonces hay distintas formas, y la práctica es social, o sea, si alguien dice "no señora, tiene que darse 10 vueltas más para volver", y levanto la ventanilla esa es una forma de dominación, haga 10 colas más. (Profesional de salud 2, entrevista presencial, junio de 2018)

Las acciones/decisiones que los/as migrantes ejercen reflejan una búsqueda constante de espacios para hacer frente a una estructura que los subordina, tanto por su origen nacional, por el color de piel, por el género y/o por la clase.

\section{CONCLUSIONES}

En el análisis se pudo ver cómo se van entretejiendo diferentes discursos y prácticas racistas, clasistas, nacionalistas y sexistas, quedando en evidencia imaginarios sociales negativos sobre ciertos colectivos migrantes residentes en la región de Aysén, que tiene efectos concretos en una discriminación sistemática en los servicios de salud. Estos imaginarios operan como marcas corporales que terminan incidiendo en sus trayectorias de vida al ser ubicados/as en espacios de subordinación social respecto de la comunidad nacional y de otros co- 
lectivos extranjeros. La construcción de la diferencia emerge institucionalmente en los dispositivos de salud a partir de la asimetría y jerarquización (Anthias, 2002).

Desde una psicología social crítica podemos cuestionar la normalización de ciertas prácticas/saberes en la intervención en salud y cómo éstas pueden recrear problemáticas sociales al establecer una relación de inclusión/exclusión según dicotomías como lo nacional/extranjero, blanquitud/negritud, mal paciente/buen paciente, buena madre/mala madre. La psicología social nos abre un horizonte para hacer una crítica a estos dualismos como construcciones sociales que inciden en las trayectorias de las personas migrantes, permitiéndonos un espacio para repensar estas mismas relaciones de poder y de subalternización entre interventores/as y personas extranjeras (Montenegro et al., 2011).

Muchas veces, la aprehensión hacia estos colectivos es explicada desde la interiorización de una identidad culturalizada e imaginada (Said, 2003) bajo una dicotomía blanquitud/negritud. Desde este posicionamiento se establece la lejanía o cercanía con las personas migrantes afrodescendientes. La principal defensa ante los efectos que podrían tener las migraciones surge en términos identitarios y fenotípicos, y es de donde emergen las justificaciones para evitar el intercambio, el distanciamiento o el rechazo, hacia ciertas personas migrantes.

Esto muestra que los grupos sociales se erigen como entidades homogéneas y compactas cultural y fenotípicamente, dejando al margen la diversidad sociocultural implícita de cualquier colectivo y la libre determinación personal. A partir de esta concepción de identidad culturalizada y racializada se consolida una distancia personal respecto de "otros/as" - subvalorados cultural y racialmente como diferentes- $y$, a la vez, una separación grupal entre "Nosotrosas/Ellos-as". Este proceso de diferenciación jerarquizado va consolidando una subordinación de los colectivos racializados al situarlos como un riesgo, peligro o en un estatus de menos valoración para la integridad de la conformación mayoritaria local o a la normatividad aceptada en la región de Aysén. Lo "normal" para las personas residentes viene marcado por la aceptación de ciertas formas culturales y sociales, lo que trae como consecuencia la generación de distintos dispositivos de control dentro de la atención sanitaria que no siempre son explícitos.

Estos obstáculos se asientan en un discurso y práctica institucional que muestra un rechazo velado hacia ciertos cuerpos racializados, reproduciendo con ello una geografía imaginaria (Said, 2003) con claras características negativas. Se observa en la cotidianidad de los sistemas de salud, cierta emergencia de un racismo sin raza (San Román, 1996); un rechazo evidente hacia ciertos colecti- 
vos, pero sin una dimensión ideológica, más bien cimentado en una lectura de la otredad bajo la alianza de características culturales, de clase y fenotípicas.

No obstante, las personas migrantes racializadas no quedan sujetas a una idea de "víctima" (Cea-Merino, 2015; Galaz y Guarderas, 2017) sino que conforman redes de apoyo que constituyen un sostén psicológico, estratégico, económico y de reproducción sociocultural que les permite desarrollar agencias frente a las discriminaciones que cruzan.

Por ello, una psicología social crítica nos invita a cuestionar el cómo se construyen "problemáticas sociales" respecto de la migración (Montenegro et al., 2011), imaginando nuevos horizontes poniendo atención a los procesos de subjetivación que se derivan de las prácticas de intervención.

\section{REFERENCIAS}

Anthias, Floya (2002). Beyond feminism and multiculturalism: locating difference and the politics of location. Women's Studies International Forum, 25(3), 275-286.

Anzaldúa, Gloria (2007). Borderlands/La Frontera: The new mestiza (3a. ed.). Aunt Lute Books. https://doi.org/10.1007/978-3-476-05728-0_4820-1

Bhavnani (Eds.), Otras inapropiables. Feminismos desde las fronteras (pp. 107-136). Traficantes de sueños.

Brah, Avtar (2013). Pensando en y a través de la interseccionalidad. En Martha Zapata, Sabina García \& Jennifer Chan (Eds.), La Interseccionalidad en Debate: Actas del Congreso Internacional "Indicadores Interseccionales y Medidas de Inclusión Social en Instituciones de Educación Superior" (pp. 14-20). MISEAL.

Cabieses, Baltica \& Oyarte, Marcela (2020). Acceso a salud en inmigrantes: identificando brechas para la protección social en salud. Revista de Saúde Pública, 54, 20. https://doi.org/10.11606/s1518-8787.2020054001501

Cáceres, Pablo (2003). Análisis cualitativo de contenido: Una alternativa metodológica alcanzable. Psico perspectivas, 2, 53-82. https://doi.org/10.5027/psicoperspectivasvol2-issue1-fulltext-3

Carrère, Cristián \& Carrère, Michelle (2015). Inmigración femenina en Chile y mercado de trabajos sexualizados: La articulación entre racismo y sexismo a partir de la interseccionalidad. Polis, 14(42), 33-52. https://dx.doi.org/10.4067/S071865682015000300003

Cea-Merino, Pastor (2015). De víctimas a agentes. Imaginarios y prácticas sobre la violencia de género en la pareja contra mujeres inmigrantes en el Estado español. Tesis doctoral sin publicar. Universidad Autónoma de Barcelona.

Cea-Merino, Pastor \& Montenegro-Martínez, Marisela (2014). Más allá de la visibilización: Problematizando discursos sobre violencia de género en la pareja contra mujeres inmigradas en España. Quaderns de Psicologia, 16(1), 167-180. https://doi.org/10.5565/rev/ qpsicologia.1194 
Chepo, Macarena; Astorga-Pinto, Sofía \& Cabieses, Baltica (2019). Atención inicial de migrantes en Chile: iniciativa en atención primaria de salud a un año de su implementación. Revista Panamericana de Salud Pública, 43(1). https://dx.doi.org/10.26633/RPSP. 2019.71

Crenshaw, Kimberlé (1989). Demarginalizing the intersection of race and sex: A black feminist critique of antidiscrimination doctrine, feminist theory and antiracist politics. University of Chicago Legal Forum.

Dube, Saurabh (2001) Sujetos subalternos. El colegio de México.

Foucault, Michael (1988). El sujeto y el poder. Revista Mexicana De Sociología, 50(3), 3-20. https://doi.org/10.2307/3540551

Foucault, Michael (1975/1992). Genealogía del Racismo. De la guerra de las Razas al Racismo del Estado. Ediciones de la Piqueta.

Galaz, Caterine (2009). Las relaciones de cooperación y exclusión entre personas con referentes culturales diversos. Un estudio sobre la alteridad. Tesis doctoral sin publicar. Universidad Autónoma de Barcelona. https://www.tdx.cat/bitstream/handle/10803/5288/cgv1de1.pdf

Galaz, Caterine \& Cea-Merino, Pastor (2020). Intervención social con migrantes en zonas extremas. Condensaciones de exclusiones múltiples desde una mirada territorial e interseccional. En Caterine Galaz, Nicolas Gissi \& Marisol Facuse (Eds), Migraciones Transnacionales: Inclusiones diferenciales y posibilidades de reconocimiento (pp. 4368). SOCIAL-EDICIONES.

Galaz, Caterine \& Guarderas, Paz (2017). La intervención psicosocial y la construcción de las “mujeres víctimas”. Una aproximación desde las experiencias de Quito (Ecuador) y Santiago (Chile). Revista de Estudios Sociales, 59, 68-82. https://doi.org/10.7440/res59.2017.06

Galaz, Caterine \& Montenegro, Marisela (2015). Gubernamentalidad y relaciones de inclusión/ exclusión: los dispositivos de intervención social dirigidos a mujeres inmigradas en España. Universitas Psychologica, 14(5), 1667-1680. https://doi.org/10.11144/javeriana.up14-5.grie

García-Dauder, Dauder (2010). Psicología y feminismo: Historia olvidada de las mujeres pioneras en psicología. Narcea

Gouveia, Marizete \& Zanello, Valeska (2019). Psicoterapia, raça e racismo no contexto brasileiro: experiências e percepções de mulheres negras. Psicologia em Estudo, 24, 1-15. https://doi.org/10.4025/psicolestud.v24i0.42738

Hill Collins, Patricia (2000). Black Feminist Thought. Routledge.

Hill Collins, Patricia \& Bilge, Sirma (2016) Interseccionality. Polity Press.

Hooks, Bell (1984). Feminist theory from margin to center. South End Press

INE-DEM (2020). Estimación de personas extranjeras residentes habituales en Chile al 31 de diciembre de 2019. INE-DEM. https: / /www.ine.cl/docs/defaultsource/demografia-y-migracion/publicaciones-y-anuarios/migraci\%C3\%B3ninternacional/estimaci\%C3\%B3n-poblaci\%C3\%B3n-extranjera-en-chile2018/estimaci\%C3\%B3n-poblaci\%C3\%B3n-extranjera-en-chile-2019metodolog\%C3\%ADa.pdf?sfvrsn=5b145256_6 
Javaloy, Federico (1994). El nuevo rostro del racismo. Anales de psicología, 10(1), 1928. https://www.um.es/analesps/v10/v10_1/04-10_1.pdf

Kalckmann, Suzana; Dos Santos, Claudete; Batista, Luis \& da Cruz, Vanessa (2007). Racismo Institucional: um desafio para a eqüidade no SUS?. Saúde e sociedade, 16(2), 146-155. https://doi.org/10.1590/s0104-12902007000200014

Kergoat, Danielle (2003). De la relación social de sexo al sujeto sexuado, Revista Mexicana de Sociología, 65(4), 841-861. https://doi.org/10.2307/3541585

Kitzinger, Celia (2003). Feminist approaches. En Clive Seale, Giampietro Gobo, Jaber Gubrium \& David Silverman (Eds.), Qualitative research practice, (25-40). Sage

Lugones, María (2008). Colonialidad y género: Hacia un feminismo descolonial. En Walter Mignolo (Comp. ), Género y descolonialidad (pp. 13-42). Ediciones del signo.

Maffesoli, Michel (2003). El imaginario Social. Anthropos, 198, 149-153.

Magliano, María (2015). Interseccionalidad y migraciones: potencialidades y desafíos. Estudos Feministas, 23(3), 691-712. http://dx.doi.org/10.1590/0104026X2015v23n3p691

McCall, Leslie (2005). The Complexity of Intersectionality. Journal of Women in Culture and Society, 30(3), 1771-1800. https://doi.org/10.1086/426800

Mohanty, Chandra (2003). Feminism without borders: Decolonizing theory, practicing solidarity. Duke University Press

Montenegro, Karla (2014). La intervención social destinada a mujeres inmigradas: un espacio de/para la configuración de diferencias y subjetividades. Athenea Digital, 14(1), 111-140. https://doi.org/10.5565/rev/athenead/v14n1.791

Montenegro, Marisela; Galaz, Caterine; Yufra, Laura \& Montenegro, Karla (2011). Dinámicas de subjetivación y diferenciación en servicios sociales para Mujeres inmigradas en la ciudad de Barcelona. Athenea Digital, 11(2), 113-132.

https://doi.org/10.5565/rev/athenead/v11n2.709

Moraga, Cherrie (2000). Loving in the war years: Lo que nunca pasó por sus labios. South End Press.

Nash, Mary (2006). Identidades de género, mecanismos de subalternidad y procesos de emancipación femenina. Revista CIDOB d'Afers Internacionals, 73-74, 39-57. http://www.jstor.org/stable/40586226.

Núñez, Nuria \& Torres, Carmen (2007). Mujeres peruanas y salud reproductiva: Usuarias de consultorios de salud de la zona norte de la Región Metropolitana. Fundación Instituto de la Mujer.

Pace, Ângela \& Lima, Marluce (2011). Racismo Institucional: apontamentos iniciais. Revista do Difere, 1(2), 1-17. https://doi.org/10.24115/S2446-6220201731223p.127$\underline{136}$

Pavón-Cuéllar, David (2019). La psicología crítica y su necesaria sensibilidad ante la violencia estructural: unaopción ante el ocultamiento psicológico del racismo y la miseria en México. En J. Ocaña Zúñiga, G. A. García Lara y O.Cruz Pérez (Coord.), Dimensiones y perspectivas de la violencia en América Latina (pp. 37-52). Grañén Porrúa y Universidad de Ciencias y Artes de Chiapas. 
Pineda G, Esther (2018). Las heridas del racismo: Efectos psicosociales de la discriminación racial en las personas afrodescendientes en América Latina. Iberoamérica Social: Revista-Red de Estudios Sociales, 7, 46-64.

https://www.aacademica.org/estherpinedag/19

Pinto Baleisan, Carolina \& Cisternas Collao, Nicole (2021). Reflexiones sobre el uso de la interseccionalidad en los estudios migratorios en Chile. Revista Punto Género, 14, 49-70. https: //doi.org/10.5354/0719-0417.2021.60866

Said, Edward (2003). Orientalismo. De Bolsillo.

Santamaría, Eduardo (2002). La incógnita del extraño. Una aproximación a la significación sociológica de la "inmigración no comunitaria". Anthropos.

Sampieri, Roberto; Collado, Carlos \& Lucio, Pilar (2004). El proceso de investigación y los enfoques cuantitativo y cualitativo: hacia un modelo integral. En Roberto Sampieri, Collado Collado \& Pilar Lucio (Eds.), Metodología de la investigación (pp. 2231). McGraw Hill Interamericana.

San Román, Teresa (1996). Los Muros de la Separación. Tecnos

Tijoux, María Emilia (2014). El Otro inmigrante “negro” y el Nosotros chileno. Un lazo cotidiano pleno de significaciones”. Boletín Onteaiken, 17, 1-15. http://onteaiken.com.ar/ver/boletin17/art-tijoux.pdf

Urzúa, Alfonso; Ferrer, Rodrigo; Olivares, Esthepany; Rojas, Jeraldinne \& Ramírez, Romy (2019). El efecto de la discriminación racial y étnica sobre la autoestima individual y colectiva según el fenotipo autoreportado en migrantes colombianos en Chile. Terapia psicológica, 37(3), 225-240. https://dx.doi.org/10.4067/S071848082019000300225

Valles, Miguel (1999). Técnicas Cualitativas de Investigación Social. Reflexión metodológica y práctica profesional. Sintesis.

Van Dijk, Teun (2006). Discurso de las élites y racismo institucional. En Manuel Lario (Coord.) Medios de comunicación e inmigración (pp. 15-36). Convivir sin racismo Programa CAM Encuentro CAM - Obra Social

Wilkinson, Sue (1991). Feminism \& Psychology: From critique to reconstruction. Feminism \& Psychology, 1(1), 5-18. https://doi.org/10.1177/0959353591011001

\section{CATERINE GALAZ}

Académica de la Universidad de Chile, es Doctora de la Universidad Autónoma de Barcelona, coordinadora del Núcleo Diversidad y Género: abordajes feministas interseccionales (UCH), participante del GT Migraciones Sur-Sur de CLACSO, de la Red Interseccionalidad, género y prácticas de resistencia $(\mathrm{UCH})$ y de la Red chilena de salud migrante (RECHISAM). 
cgalazvalderrama@uchile.cl

https://orcid.org/0000-0001-6301-7609

\section{PASTOR CEA}

Académico del Departamento de Ciencias Sociales de la Universidad de Aysén. Psicólogo, Máster de Intervención Psicosocial y Doctor en Psicología Social por la Universidad Autónoma de Barcelona. Sus actuales líneas de estudio se vinculan con los procesos migratorios transnacionales y los procesos de intervención psicosocial.

pastor.cea@uaysen.cl

https://orcid.org/0000-0002-3797-1711

\section{DANIELA MOLINA}

Trabajadora Social de la Universidad de Chile. Investigadora en Núcleo I+D Relaciones socioeconómicas y luchas sociales y Núcleo de Diversidad y Género: Abordajes Feministas Interseccionales y del Núcleo Territorio y Diversidad (2017-2020).

daniela.molina.n@ug.uchile.cl

https://orcid.org/0000-0002-7772-7625

\section{DANIELA CASTRO}

Trabajadora Social de la Universidad de Chile, Tesista en Red Interseccionalidad, género y prácticas de resistencias $(\mathrm{UCH})$ en Magíster en Psicología Comunitaria (UCH), Investigadora en Núcleo I+D Relaciones socioeconómicas y luchas sociales, Diversidad y Género: Abordajes Feministas Interseccionales y Territorio y Diversidad (2017-2020), Participante Comisión Octubre 2019.

daniela.p. castro.reyes@gmail.com

https://orcid.org/0000-0002-5711-2754

\section{MARÍA JOSÉ ORTEGA}

Trabajadora Social Universidad de Chile, Diplomada en Psicología Social y Procesos Políticos (FACSO- UCH). Investigadora en Núcleo I+D Relaciones socioeconómicas y luchas sociales, el Núcleo Diversidad y Género: Abordajes Feministas Interseccionales y el Núcleo Territorio y Diversidad (2017-2020).

mjortega26@gmail.com

https://orcid.org/0000-0002-6994-3117

\section{AGRADECIMIENTOS}

Al Núcleo I+D Diversidad y Género de Trabajo Social de la Universidad de Chile y a la carrera de Trabajo Social y al Fondo Semilla de la Universidad de Aysén.

\section{FORMATO DE CITACIÓN}

Galaz, Caterine; Cea, Pastor; Molina, Daniela; Castro, Daniela \& Ortega, María José (2021). Una mirada interseccional a las prácticas de salud en Aysén. Procesos de racialización en Chile. Quaderns de Psicologia, 23(3), e1750.

https: / /doi.org/10.5565/rev/qpsicologia.1750 


\section{HISTORIA EDITORIAL}

Recibido: 18-01-2021

$1^{\text {a }}$ revisión: 12-04-2021

Aceptado: 26-04-2021

Publicado: 25-01-2022 